\title{
Some Thoughts on Using Biochar to Improve Albic Soil
}

\author{
Dawei Yin 1,2, Xiaohong Guo', Jiabo Wang1, Yingwen Xue, Menghong Liư ${ }^{1}$, Hongyu Li ${ }^{1}{ }^{*}$ \\ ${ }^{1}$ Agricultural College, Heilongjiang Bayi Agricultural University, Daqing, China \\ ${ }^{2}$ Agricultural College, Shenyang Agricultural University, Shenyang, China \\ Email: yindazhiyindawei@126.com, *178428592@qq.com.
}

How to cite this paper: Yin, D.W., Guo, X.H., Wang, J.B., Xue, Y.W., Liu, M.H. and Li, H.Y. (2019) Some Thoughts on Using Biochar to Improve Albic Soil. Agricultural Sciences, 10, 807-818.

https://doi.org/10.4236/as.2019.106062

Received: June 4, 2019

Accepted: June 25, 2019

Published: June 28, 2019

Copyright $\odot 2019$ by author(s) and Scientific Research Publishing Inc. This work is licensed under the Creative Commons Attribution International License (CC BY 4.0).

http://creativecommons.org/licenses/by/4.0/

\begin{abstract}
Biochar of agricultural and forestry waste is one of the core ways to solve the greenhouse effect, and it is also the key measure to improve the land productivity of China's low yield fields and realize the sustainable development of agriculture. Due to the large area of China's albic soil, the long-term drought and flood disasters make it a typical low-yield field, which has not been effectively treated. Biochar provides new hope for improving low-yield albic soil. This paper introduces the basic properties of biochar, the albic soil formation mechanism, the low yield of albic soil factors and the improving theoretical basis of biochar. The paper introduces the technical features of the albic soil improvement and the advantages of the albic soil improvement by biochar. In the view of ascending low production potential, solving the greenhouse effect, achieving agricultural sustainable development, the application value and development prospects of albic soil improved by biochar are discussed.
\end{abstract}

\section{Keywords}

Biochar, White Paper Clay, Food Security, Sustainable Development

\section{Introduction}

Biochar is recognized as an emerging technology to ensure human food, environment and energy security under high pressure in the new century. The development of biochar industry in China is based on the utilization of agricultural and forestry wastes, focusing on solving non-point source pollution in rural areas, improving the quality of cultivated land, promoting carbon sequestration and emission reduction, and realizing sustainable agricultural development. Around the deep field of agriculture, domestic and foreign researchers have preliminarily confirmed that biochar can effectively improve soil structure, physical chemistry properties and biological properties, increase crop yield, control envi- 
ronmental pollution, increase "agricultural carbon sink" and reduce greenhouse gas emissions. Biochar can be used in the field, which builds a bridge between agricultural and forestry wastes such as straw with cultivated land. Biochar builds a benign model of high yield, low carbon and sustainable development of agriculture.

China's food security is controlled by the actual grain production capacity of $70 \%$ of medium-low yield fields. Albic soil is a kind of soil formed through albic formation process with dark humus surface layer, off-white sub-surface layer, and dark brown adhesive deposition layer. The albic soil of its profile configuration is Ah-E-Bt-C [1]. The United Nations Food and Agriculture Organization reports that there are 32 countries and regions in the world where albic soil is distributed. Albic soil mainly is distributed in the northeast region of China. The albic soil was mainly in Jiangsu, Shandong, Anhui, Henan and Sichuan provinces, and the total areas of albic soil were 5.272 million.hm². The albic horizon of albic soil led to the drought and flood disasters for a long time. The albic soil becomes the typical middle- and low-yielding fields, adding serious soil and water loss, bringing the ecological environment irreversible malignant effect [1]. The positive effects of biochar on agriculture and environment will become a new force to improve the grain potential and a choice to cope with China's national conditions.

This paper briefly introduces the basic properties of biochar and albic soil formation mechanism, analyzes the low yield factors of albic soil and biochar improvement. The more classes of technical features for improving the albic soil were analyzed, and the advantage of albic soil improvement by biochar was analyzed. In the view of the low-yielding fields of grain in ascension potential, solving the greenhouse effect, and realizing agricultural sustainable development, the biochar application value and development prospects to improve low yield albic soil are discussed.

\section{Basic Properties of Biochar}

Biochar generally refers to the pyrolysis product of biomass under the conditions of hypoxia and relatively low temperature $\left(<700^{\circ} \mathrm{C}\right)$. It is mainly composed of aromatic hydrocarbon, elemental carbon or carbon with graphite structure and contains more than $60 \%$ carbon elements [2]. Biochar has rich porous structure, huge specific surface area, and strong adsorption capacity which can increase soil porosity, lower bulk density and water-holding performance. The biochar can improve soil quality, enhance the nutrient contents, promote formation and organic matter, promote microorganism growth, and promote interaction by improving soil physic-chemical and biological properties of soil [3].

\section{The Section Composition and Formation Mechanism of Albic Soil}

The albic soil consists of four layers. The layers are black soil layer, albic horizon 
layer, sedimentary layer and parent material layer from top to bottom. The albic soil was formed in the area with a gradient of about $1^{\circ}-3^{\circ}$, and its formation was closely related to the parent material. The parent material of Northeast of China albic soil is mainly quaternary river and lake clay sediments. The clay particles in the parent material are easy to be suspended and dispersed, and they are mechanically leached with the descending water, making the upper layer silty and the lower layer sticky. This is the primary stage of Northeast China albic soil formation. With the growth of plants and accumulation of organic matter, suspended migration of clay particles is inhibited, mainly resulting in reductive leaching and complexation leaching of metal ions. The non-ferrous metal ions exude from the soil or oxidize to form iron and manganese nodules, which finally form decolorized albic horizon layer and sticky sediment layer. However, the albic horizon layer in Huaibei region is formed by residual clay of parent material, and the albic horizon layer is caused by "double parent material" [4].

\section{Barrier Factors of Albic Soil and Improvement Basis of Biochar}

The dense layer of albic soil leads to the long-term occurrence of drought and flood, making albic soil a typical low-yield field. The key to control albic soil is to improve the physical, chemical and biological properties of albic horizon, and to change the albic horizon from a dense layer to a loose soil area where crops can grow. Biochar has good structural characteristics and nutrient quantity, and can exert long-term positive effects on the physical chemistry and biological properties of soil.

\subsection{Dense Structure, Bulk Density and Hardness of the Albic Hozion}

The albic horizon has a dense structure, with sand content of $79.12 \%-80.60 \%$ and clay content of $19.4 \%-20.88 \%$ [1]. The volume ratio of the sand content and clay content is close to the ideal ratio of the densiest filling. The bulk density of the albic horizon was up to $1.40 \mathrm{~g} \cdot \mathrm{cm}^{-3}-1.61 \mathrm{~g} \cdot \mathrm{cm}^{-3}$ and the hardness was up to $40-50 \mathrm{~kg} \cdot \mathrm{cm}^{-2}$. The roots could hardly penetrate the soil. Particle size and density of biochar are determined by the nature of raw materials and pyrolysis process [5]. Biochar can change the mechanical composition of soil, which is related to the size range of biochar from micron to centimeter. The density of biochar is low, with the solid density ranging from 1.5 to $2.1 \mathrm{~g} \cdot \mathrm{cm}^{-3}$ and the volumetric density ranging from 0.05 to $0.5 \mathrm{~g} \cdot \mathrm{cm}^{-3}$, which can effectively reduce the bulk density of soil [2] [3].

\subsection{The Total Porosity of the Albic Horizon Is Low}

The total porosity of the albic horizon was lower than $50 \%$, mainly with small pores (Diameter $<0.0002 \mathrm{~mm}$ ), up to $21.1 \%-23.5 \%$, and the content of large pores $(>0.05 \mathrm{~mm})$ was only $1.3 \%-1.7 \%$. The low total porosity of the albic horizon inhibits water infiltration, root growth, microbial reproduction and other 
processes [6]. Biochar has a good physical structure. The soil porosity can be improved by means of changing soil mechanical composition, promoting agglomeration formation and enhancing root growth by biochar [7].

\subsection{The Aggregate Quantity of Albic Soil Is Low}

The content of water-stable aggregates in the surface layer of albic soil is as low as $8 \%$, while there is no water-stable aggregates in the albic horizon [6]. Biochar is rich in carbohydrates, long-chain olefin and other organic macromolecules, and can also promote the secretion of microbial polysaccharides(such as Glomalin-globulin). Polysaccharides are important substances for the formation and stability of soil aggregates. Biochar directly and indirectly promotes the formation of organic-inorganic complexes and soil aggregates.

\subsection{The Poor Water Permeability of Albic Horizon}

The slope of albic soil is relatively slow, ranging from $1^{\circ}$ to $3^{\circ}$. The water permeability coefficient of albic horizon is very low about $0.20 \mathrm{~mm} \cdot \mathrm{min}^{-1}$. In the period of concentrated rainfall, the rainwater is not easy to seep out in the side of the surface layer, nor to penetrate into the bottom soil, causing serious flooding. Biochar can effectively increase soil porosity and water permeability [8].

\subsection{The Moisture Content of Albic Soil Is Low}

The water content of the albic horizon is significantly lower than that of the surface layer and deposition layer, which is due to the low porosity of the albic horizon, the water in the surface layer is difficult to penetrate into the albic horizon, and the rise of water in the sedimentary layer is also restrained. When the albic soil is in the dry period, the albic horizon cannot effectively supply water to the surface layer [8]. The surface oxidized biochar has the property of water retention, which is due to its large specific surface area (about $300 \mathrm{~m}^{2} \cdot \mathrm{g}^{-1}$ ) and abundant hydrophilic groups [9].

\subsection{The Nutrient Deficiency of Albic Horizon}

The nutrient deficiency of the albic horizon is due to the dense and compact structure, which inhibits the reproduction of microorganisms, the growth of roots and the migration of nutrients from the surface layer to the bottom layer. Biochar can directly and indirectly regulate soil nutrient content in a positive way. Biochar can provide a considerable amount of nutrients. Biochar its porous structure, huge specific surface area and charge density can adsorb exchangeable nutrient ions and indirectly improve the content of soil nutrients. Biochar can also promote the aggregation of adsorbed organic molecules to form soil organic matter through surface catalytic activity [10].

\subsection{The Low Number of Microorganisms in the Albic Horizon}

The number of microorganisms in the albic horizon is much lower than the sur- 
face layer, because the dense structure of the albic horizon cannot provide good breeding space for microorganisms, and the low content of organic matter also inhibits the reproduction of microorganisms. Biochar can increase the variety and quantity of soil microorganisms. A large number of micropores are distributed on the surface of biochar, which provide a good "refuge" place for microbial habitat and reproduction avoiding the impact of microbial invasion and dehydration, reducing the survival competition among microorganisms. Biochar contains materials of different kinds and components, which can provide sufficient nutrients for the growth of microorganisms. Biochar promotes the respiratory metabolic process of microbial communities and the ability of microorganisms to use substrates by increasing soil $\mathrm{pH}$. When the $\mathrm{pH}$ is from 3.7 to 8.3, the number of microorganisms increases [11].

\subsection{The Albic Soils Are Acidic}

The acidity of albic soil is related to the soil parent material, organic acid secreted by plant, and the application of physiological acid fertilizer. Biochar is usually alkaline with a $\mathrm{pH}$ range of $8.2-13.0$, which is related to the pyrolysis of raw material organic acid and generation of ash in the preparation process of biochar. The $\mathrm{pH}$ value of biochar produced under high mineral ash content and high temperature is relatively high. The biochar contains a large number of salt ions, which increase the soil $\mathrm{pH}$ value by reducing the content of exchangeable hydrogen ions and exchangeable aluminum ions in the soil [12].

\subsection{The Low Susceptibility of Albic Soil}

High soil magnetization indicates good maturation degree and high fertility, and the magnetization of albic soil is as low as $6 \times 10^{-6}-13 \times 10^{-6}$ CGMS. The magnetization ratio of the albic layer is lower than that of the surface layer and the sedimentary layer, and the albic horizon shows a CGMS "concave" shape in the soil profile, which is because albic soil underwent a strong leaching process in the soil formation, forming an albic horizonr with poor iron and manganese [13].

\section{Improvement Technology of Albic Soil}

\subsection{Biological and Organic Materials Are Used to Improve Albic Soil}

Early albic soil improvement focused on organic soil improvement. According to the results of many years' experiments in the coastal region of Russia, the yield of wheat increased by $30 \%-105 \%$, soybean increased by $25 \%-80 \%$ and sugar beet increased by $35 \%-100 \%$ in the first year after applying $36-41 \mathrm{t} \cdot \mathrm{hm}^{-2}$ manure on the albic soil, and the fertilizer yield increased by 3 - 4 years. The results of long-term positioning experiment of Heilongjiang Bayi Agricultural University show that the content of soil organic matter, total nitrogen, available nitrogen and available phosphorus increase significantly after 6 consecutive 
years of applying organic fertilizer and straw to the field. In addition, the research on using green manure to improve the soil surface layer has a history of many years in China, including variety selection, utilization technology and fertilizer efficiency test of green manure, etc. [14]. However, these techniques only improve the physical and chemical properties of the albic soil surface layer, but rarely touch the barrier layer. On the other hand, green fertilizer and organic fertilizer technology have long cycle, high cost and slow effect. In addition, the distribution area of albic soil is mostly vast and sparsely populated. Therefore, soil improvement methods such as organic fertilizer are restricted by insufficient fertilizer sources and inconvenient transportation, it is difficult to popularize them in large areas.

\subsection{Sand Mixing Is Used to Improve the Albic Soil}

Sand mixing measures can change the mechanical composition and increase the porosity and moisture content of the albic soil. Sand consumption is determined according to the mechanical composition of the albic horizon by Mudanjiang agricultural science and technology institute. The research results show that 10 cubic meters of sand per mu can be applied continuously for 2 - 3 years for improvement. However, the content of organic matter in sand is low, which is not conducive to the formation of aggregates [15].

\subsection{Improvement with Lime}

Albic soil is a weak acid soil, and the surface layer $\mathrm{pH}$ of 1:2.5 $\left(\mathrm{H}_{2} \mathrm{O}\right)$ is 5.5. In the 853 farm of Heilongjiang state bureau of agricultural reclamation, lime was used to improve the surface layer of albic soil. The experimental results showed that the yield of soybean increased by $18.9 \%$ in the first year, $5.9 \%$ in the second year, $11.6 \%$ in the third year and $11.5 \%$ in the fourth year. In our country the Ussuri River across the Russian coastal agricultural reclamation experimental station of lime ameliorant albic soil do a deep research into the soil, the experimental station in Dmitry, state farms of albic soil rotation positioning tests have been carried out, the results show that under the condition of without chemical fertilizers, with the increase of the dosage of lime, there are increased crop yields, and increase rate of $9.7 \%-35.3 \%$ [14]. But this technique still does not reach the barrier layer of white pulp.

\subsection{Mechanical Improvement of Albic Soil}

In order to break the albic horizon and thicken the active soil layer, deep turning and subsoiling improvement machinery came into being. In order to break the soil hard horizon, deep tillage test was carried out on the albic horizon, and soil operations with tillage depth of more than $25 \mathrm{~cm}$ were collectively referred to as profile improvement technology. The early mechanical improvement measures in China are deep soil turning. However, practice has proved that blindly deepening the tillage layer and mixing the barren albic horizon into the surface layer 
can lead to the degradation of soil physical and chemical properties in the tillage layer and the decline of crop yield, under the circumstance that the increase of organic fertilizer cannot be guaranteed. Therefore, the improved albic horizon technology of deep scarification was popularized in production. The advantage of deep scarification is that albic horizon can be broken without destroying the order of albic soil layers, which can not only loosen albic horizon, but also maintain the fertility of surface soil. However, the aftereffect of soil improvement is usually only one year, and the agricultural producers in the albic soil areas have to pay a high cost for the annual deep pine. According to the investigation, the reason why the above-mentioned mechanical soil improvement technology has a short after-effect duration is mainly because of the high content of powdery particles in the albic horizon and the strong resilience of the soil. Proved through artificial simulation test, that the adverse effects of the albic horizon can be completely eliminated by mixing the albic horizonr and sedimentary layer in a certain proportion, under the condition that the fertile surface layer of the soil remains unchanged [15] [16] [17].

\subsection{Improvement of Core Soil and Fertilizer}

Core soil cultivation refers to the operation to achieve the purpose of core soil cultivation by putting soil improving materials into the core soil. According to the mechanical characteristics of "two-layer" (that is, the mechanical compositions of the albic horizon and the sedimentary layer are different), mixing the albic horion and the sedimentary layer can improve the poor physical properties of the albic horizon. The three-stage core soil mixed layer plough obviously increased crop yield, after core layer mixing. In order to increase nutrients in the albic horizon, experiments were carried out successively to cultivate fertilizer in the core soil. However, when the albic horizon is mixed with the sediment layer, the soil quality is still sticky due to the lack of large-sized soil particles [18].

All the methods mentioned above have some effect on the improvement of albic soil, but due to many reasons, the low yield albic soil in China has not been effectively treated for a long time.

\subsection{Use Biochar to Improve Albic Soil}

Due to its inherent structural and physico-chemical characteristics, biochar has an impact on soil bulk density, water content, porosity, cation exchange capacity and nutrient content after it is applied to the soil, thus directly or indirectly affecting the soil micro-ecological environment. Therefore, biochar has a theoretical basis for improving the albic horizon. We begin from 2011 in Heilongjiang province agricultural reclamation administration, and launched albic soil improvement long-term trial field by biochar in the 850 farm. The preliminary results showed that biochar can effectively improve the albic hortizon physical and chemical properties, improve the albic horizon microorganism quantity and enzyme activity, a significant increase in the number of roots in the maize yield of 
albic horizon, effectively eliminate the albic horizon frequent soil drought and flood disaster, but so far of biochar on the obstacle of low yield albic soil mechanism has not been completely elucidated.

The above $4.1-4.5$ techniques have a positive effect on the improvement of clay. However, the existing problems are that the physical structure of the improved white pulp layer is still dense and sticky, or it involves a huge amount of soil improvement works and special supporting soil improvement machinery, which is very difficult to promote and apply. There is an urgent need for a new type of improved clay model which can be easily operated by large machinery in production.

\section{Analysis of the Advantages and Disadvantages of Biochar Modified Albic Soil}

China's biochar industry is based on the utilization of agricultural and forestry waste resources. It is a "bridge" between abandoned agricultural resources and soil. It is the core mode of realizing agricultural cycle, low carbon and sustainable development. Biochar has the following advantages:

\subsection{Biochar Is Feasible to Apply into the Albic Horizon through Deep Pine Machinery}

Biochar has a granular structure and can be easily applied into the albic horizon by deep pine machinery, which has the feasibility of large-scale popularization. In contrast, the size of crop straw is huge, which cannot be applied into the albic horizon by existing large machinery, and is only limited to the scope of theoretical research.

\subsection{Biochar Has Wide Sources and Low Cost}

Crop straw, forestry waste, livestock and poultry waste and other renewable resources can be used as raw materials for biochar, which has a wide range of sources and low cost. Biochar can be sourced locally, carbonized and returned to the field, saving transportation cost.

\subsection{The Effect of Biochar on Physicochemical and Biological Properties Has a Long-Term Effect}

Biochar can change soil mechanical composition, increase porosity, and promote the combination with soil particles and aggregate structure by relying on the abundant functional groups on the surface. Biochar can adsorb nutrient ions through the rich chemical bonds on the surface, which helps to reduce nutrient loss and release nutrient ions slowly. The abundant microporous structure of biochar is the "habitat" of microorganisms, which contains abundant carbon source and provides continuous supply to microorganisms. Biochar makes use of its good physical structure to maintain the durability of the improvement effect by improving the physicochemical and biological properties. 


\subsection{Biochar Has Built a Circular, Low-Carbon and Sustainable Agricultural Model}

With the application of biomass carbonization to farmland technology, carbon equivalent to about $50 \%$ of biomass can be stored in the soil for a long time and become a net sink of carbon. This method of "taking from the field" and "applying to the field" promotes the internal circulation of the matter in the agricultural system, and constructs an agricultural mode of "recycling", "low-carbon" and "sustainable development" that is suitable for China's national conditions in the new century.

\subsection{Biochar Is Expensive}

At present, the cost of biochar is relatively high, which is a problem in the process of improving the soil quality. How to further reduce the cost of biochar is a problem that needs further research in the future.

\section{Outlook}

Improving the grain potential of low-and medium-yield fields will be the key to ensuring national food security in the future. In 2013, China's grain output increased for ten consecutive years, exceeding 600 million tons. It is estimated that by 2030 , China's population will reach 1.65 billion, and the demand for grain will be about 759 million tons. Currently, China's cultivated land area is 1.83 billion mu. With the rapid progress of urbanization and infrastructure construction, China's cultivated land area is expected to maintain the red line of 1.8 billion mu in 2020 under the constraints of the cultivated land protection system. With the increase of population and the shortage of cultivated land, we are facing more severe food pressure, which requires us to focus on improving the fertility of medium- and low-yielding fields and increase the yield per unit area. The area of medium and low-yielding fields in China shows an increasing trend, accounting for $70 \%$ of the total cultivated land area in the "eleventh five-year plan" period, and $7.70 \%$ more than that in the "tenth five-year plan" period. Assuming that all the medium and low-yielding fields in China are improved, the total grain will increase by $0.62-123$ million tons. Biochar is a "precious" soil conditioner. Thousands of years ago, Indian ancestors used biochar to improve the poor and highly weathered eluvial soils in the amazon basin, which is still one of the most fertile soils in the world, which is the most powerful proof of the positive effect of biochar on poor soils [19]. Albic soil accounted for $4.5 \%$ of lowand medium-yield fields. The author confirmed that biochar turned albic horizon into the appropriate crop growth layer which solved the problem of drought and flood disasters, and the yield for biochar treatment increased $19.49 \%$ $37.64 \%$ than control. China will increase grain output by 7.9095 million if the albic soil is all modified.

Biochar will become the core pillar to solve global warming and realize China's low-carbon economic development model. Global carbon dioxide emissions 
hit a record high of 36 billion tons in 2013, 2.1 percent higher than 2012, including about 10 billion tons in China, according to data released by the global carbon plan (GCP). The Copenhagen accord calls for global temperature increases to be limited to $2^{\circ} \mathrm{C}$, to be achieved only when $\mathrm{CO}_{2}$ emissions peak in 2015 and then decline. According to the work plan for controlling greenhouse gas emissions issued by the state council during the 12th five-year plan period, carbon dioxide emissions per unit of GDP will drop by 17\% in 2015 compared with 2010. In the face of severe international and domestic situation, biochar provides a key feasible way for carbon sequestration and emission reduction [20]. Biochar itself is a net "negative carbon" process. After the carbonization of 1 ton of agricultural and forestry waste, it is equivalent to the assimilation of about $0.5 \mathrm{t} \mathrm{CO}_{2}$. When applied to soil, it can also reduce greenhouse gas emissions such as $\mathrm{N}_{2} \mathrm{O}$ and $\mathrm{CH}_{4}$. China's annual output of agricultural and forestry waste is about 1.4 billion $\mathrm{t}$, making biochar equivalent to reducing $\mathrm{CO}_{2}$ emissions by 700 million t. Ensuring national food security, tackling the greenhouse effect and maintaining ecological balance are the key tasks. We firmly believe that biochar will be a "hero" to solve the bottleneck problem.

\section{Acknowledgements}

In this paper, the national 13th five-year key research and development plan "biochar based fertilizer replacement technology integration and model construction" (2017YFD0200803); National key research and development plan of the 13th five-year plan "the formation mechanism of differences in japonica rice yield and efficiency levels in northeast China and the ways of increasing yield and efficiency" (2016YFD0300104); National science and technology support program (2015BAD23b05-04); Natural science foundation of Heilongjiang province (QC2017023); Key project of natural science foundation of Heilongjiang province (ZD2017008); Heilongjiang postdoctoral funding project (LBH-Z17186); National college students innovation and entrepreneurship project (201810223019); Research team platform support plan of Heilongjiang Bayi Agricultural University (TDJH201802); Heilongjiang state bureau of agricultural reclamation project (HNK135-02-02); Heilongjiang state bureau of agricultural reclamation science and technology program (HNK135-02-03).

\section{Conflicts of Interest}

The authors declare no conflicts of interest regarding the publication of this paper.

\section{References}

[1] Zhu, B.G., Zhang, C.F. and Jia, H.B. (2014) Effect of Subsoil Mixing Interval on Physical and Chemical Characteristics and Yield of Crops in Plano Soil. Chinese Journal of Soil Science, 45, 704-710.

[2] Ozcimen, D. and Karaosmanoglu, F. (2004) Production and Characterization of 
Bio-Oil and Biochar from Rapeseed Cake. Renewable Energy, 29, 779-787. https://doi.org/10.1016/j.renene.2003.09.006

[3] Chan, K.Y., Zwieten, V.L. and Meszaros, I. (2007) Agronomic Value of Greenwaste Biochar as a Soil Amendment. Australian Journal of Soil, 45, 629-634. https://doi.org/10.1071/SR07109

[4] Zhu, B.G., Zhang, C.F. and Jia, H.B. (2018) Improving Effect of Planosol by Deep Tillage Combined with Subsoil Mixed with Ameliorative Materials. Transactions of the Chinese Society of Agricultural Engineering, 34, 107-114.

[5] Liu, T.T., Gao, Z.C., Kuang, E.J., et al. (2012) Study on Physical Characteristics of Planosol I. Some Physical Characteristics about Soil Compaction and Permeability. Heilongiiang Agricultural Sciences, 4, 49-52. (In Chinese)

[6] Liu, T.T., Gao, Z.C., Kuang, E.J., et al. (2012) Study on Physical Characteristics of Planosol III. Microstructure and Porosity Features. Heilongjiang Agricultural Sciences, 6, 45-48. (In Chinese)

[7] Tian, J., Wang, J., Dippold, M., et al. (2016) Biochar Affects Soil Organic Matter Cycling and Microbial Functions But Does Not Alter Microbial Community Structure in a Paddy Soil. Science of the Total Environment, 556, 89-97. https://doi.org/10.1016/j.scitotenv.2016.03.010

[8] Tian, X.P. and Zhang, Z.Y. (2015) Study on pH Variation under Different Tillage System in Ablic Soil. Journal of Northeast Agricultural University, 46, 37-40. (In Chinese)

[9] Wang, J., Xiong, Z., Kuzyakov, Y., et al. (2016) Biochar Stability in Soil: Meta-Analysis of Decomposition and Priming Effects. Global Change Biology Bioenergy, 8, 512-523. https://doi.org/10.1111/gcbb.12266

[10] Wu, M., Han, X., Zhong, T., et al. (2016) Soil Organic Carbon Content Affects the Stability of Biochar in Paddy Soil. Agriculture, Ecosystems and Environment, 223, 59-66. https://doi.org/10.1016/j.agee.2016.02.033

[11] Zhou, Y., Berruti, F., Greenhalf, C., et al. (2017) Increased Retention of Soil Nitrogen over Winter by Biochar Application: Implications of Biochar Pyrolysis Temperature for Plant Nitrogen Availability. Agriculture, Ecosystems and Environment, 236, 61-68. https://doi.org/10.1016/j.agee.2016.11.011

[12] Mickan, B.S., Abbott, L.K., Stefanova, K., et al. (2016) Interactions between Biochar and Mycorrhizal Fungi in a Water-Stressed Agricultural Soil. Mycorrhiza, 26, 565-574. https://doi.org/10.1007/s00572-016-0693-4

[13] Huo, Y.P., Liu, J.X. and Zhang, H. (1983) Discussion on the Problem of Planosol Improvement Based on the Study of Its Physical Property of Moisture. Journal of Northeast Agricultural College, 3, 69-75. (In Chinese)

[14] Kuang, E.J., Liu, F. and Gao, Z.C. (2011) Research on Subsoil Fertilization of Albic Luvisol Improvement II. The Contribution of Subsoil on the Crop Yield and Effect of Subsoil Fertilization with P and Ca. Chinese Journal of Soil Science, 42, 953-957.

[15] Zhao, D.L., Liu, F. and Jia, H.B. (1994) Study on the Effect of Mixed Layer Tillage on Improving Mortar Soil. Chinese Agricultural Science, 27, 37-44.

[16] Duan, X.W., Xie, Y., Liu, B.Y., et al. (2012) Soil Loss Tolerance in the Black Soil Region of Northeast China. Journal of Geographical Sciences, 22, 737-751. https://doi.org/10.1007/s11442-012-0959-5

[17] Gong, H.L., Meng, D, Li, X.J., et al. (2013) Soil Degradation and Food Security Coupled with Global Climate Change in Northeastern China. Journal of Geographical Sciences, 23, 562-573. https://doi.org/10.1007/s11769-013-0626-5 
[18] Liu, F., Zhang, Y.L. and Jia, H.B. (2001) Three-Stage Subsoil Mixing Plough and Its Improvement of Planosol. Transactions of the Chinese Society of Agricultural Engineering, 3, 57-61. (In Chinese)

[19] Chen, W.F., Zhang, W.M. and Meng, J. (2013) Advances and Prospects in Research of Biochar Utilization in Agriculture. Chinese Journal of Soil Science, 46, 3324-3333. (In Chinese)

[20] Meng, J., Zhang, W.M., Wang, S.B., et al. (2011) Development and Prospect of Carbonization and Returning Technology of Agro-Forestry Residue. Journal of Shenyang Agricultural University, 42, 387-392. (In Chinese) 